\title{
The Visual System \\ from Genesis to Maturity
}




\section{The Visual System from Genesis to Maturity}

\section{Roberto Lent}

Editor

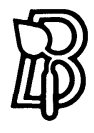

Springer Science+Business Media, LLC 


\author{
Roberto Lent \\ Departamento de Neurobiologia \\ Instituto de Biofísica Carlos Chagas Filho \\ Universidade Federal do Rio de Janeiro \\ Centro de Ciências de Saúde, Bloco G \\ Cidade Universitária \\ 21941 Rio de Janeiro R.J. \\ Brazil
}

Library of Congress Cataloging-in-Publication Data

The Visual system from genesis to maturity / edited by Roberto Lent.

p. $\mathrm{cm}$.

Includes bibliographical references and index.

ISBN 978-1-4899-6728-2 -X (H : acid-free). - ISBN 978-1-4899-6728-2-X

1. Eye-Differentiation. 2. Eye-Growth. 3. Visual pathways-

-Differentiation. 4. Visual pathways-Growth. I. Lent, Roberto.

QP475.V63 1992

$599^{\prime} .01823-\mathrm{dc} 20$

CIP

Printed on acid-free paper.

(C) Springer Science+Business Media New York 1992

Originally published by Birkhäuser Boston in 1992

Softcover reprint of the hardcover 1st edition 1992

Copyright is not claimed for works of U.S. Government employees.

All rights reserved. No part of this publication may be reproduced, stored in a retrieval system or transmitted, in any form or by any means, electronic, mechanical, photocopying, recording or otherwise, without prior permission of the copyright owner.

Permission to photocopy for internal or personal use, or the internal or personal use of specific clients, is granted by Birkhäuser Boston for libraries and other users registered with the Copyright Clearance Center (CCC), provided that the base fee of $\$ 5.00$ per copy, plus $\$ 0.20$ per page is paid directly to CCC, 21 Congress Street, Salem, MA 01970, U.S.A. Special requests should be addressed directly to Springer Science+Business Media, LLC.

ISBN 978-1-4899-6728-2 ISBN 978-1-4899-6726-8 (eBook)

DOI $10.1007 / 978-1-4899-6726-8$

Typeset by ATLIS Graphics

987654321

Cover: A detail of "Solomon's Justice", by the Brazilian painter Candido Portinari (1903-1962), showing an ambiguous figure.

Art Museum of São Paulo (MASP). Reproduced by permission of the Portinari Project. 


\section{Contents}



An Overview-Development of the Primate Visual System: From Photoreceptors to Cortical Modules

Pasko Rakic ...............................................................

\section{SECTION 1-GENESIS}

1. Cellular and Molecular Mechanisms Regulating Retinal Cell Differentiation

Ruben Adler

2. Differentiation of the GABAergic System in the Avian Retina: Control of Glutamic Acid Decarboxylase Expression by GABA Fernando G. De Mello, Jan N. Hokoç, Ana L. M. Ventura, and Patrícia F. Gardino

3. Role of Acetylated Gangliosides on Neuronal Migration and Axonal Outgrowth

Rosalia Mendez-Otero, Burkhard Schlosshauer, and Martha Constantine-Paton

4. The Activity-Dependent Mechanism in the Development of the Refined Retinotopic Map

Hollis T. Cline

5. Mechanisms of Dendritic Tree Development in Mammalian Retinal Ganglion Cells Ary S. Ramoa and Edna N. Yamasaki

6. Dendritic Competition: A Principle of Retinal Development Rafael Linden

7. Role of Postsynaptic Activity in Retinogeniculate Pattern Formation Mriganka Sur, Jong-On Hahm, and Manuel Esguerra

8. Generation of Cell Diversity in the Mammalian Visual Cortex Marla B. Luskin 
9. Different Developmental Strategies of the Telencephalic Commissures:

A Comparison between the Ontogeneses of Visual Callosal Connections and of Olfactory Commissural Connections in Rodents Roberto Lent

\section{SECTION 2-MATURITY}

10. Organization of Catecholaminergic Amacrine Cells in the Rhesus Monkey Retina

Andrew P. Mariani and Jan Nora Hokoç

11. Neurotransmitter Drugs that Affect Vertebrate Eye Movements Michael Ariel

12. Evidence for Commissural Interactions in the Nuclei of the Optic Tract of the Opossum Eliane Volchan, Antônio Pereira, Jr., Raymundo Francisco Bernardes, and Carlos Eduardo Rocha-Miranda

13. A Comparative Survey of Magnification Factor in V1 and Retinal Ganglion Cell Topography of Lateral-Eyed Mammals

Cristovam W. Picanço-Diniz, Luiz Carlos L. Silveira, and Eduardo Oswaldo-Cruz

14. Columnar Organization of Primate Visual Cortex Roger B. H. Tootell and Richard T. Born

15. In Search of the Canonical Microcircuits of Neocortex

Rodney J. Douglas and Kevan A. C. Martin

16. Visual Responses Outside the Classical Receptive Field in Primate Striate Cortex: A Possible Correlate of Perceptual Completion R. Gattass, M. Fiorani, Jr., M.G.P. Rosa, M.C.G. Piñon, A.P.B. Sousa, and J.G.M. Soares

17. Inferior Temporal Cortex: Neuronal Properties and Connections in Adult and Infant Macaques

Charles G. Gross and Hillary R. Rodman

18. Reorienting Visual Spatial Attention: Is It Based on Cartesian Coordinates?

Luiz Gawryszewski, Rosalia B. Faria, Tania G. Thomaz, Walter M. Pinheiro, Giacomo Rizzolatti, and Carlo Umiltà 


\section{Preface}

I must confess openly, before presenting this book to the reader, that the task of producing it was much more enjoyable to me than I had suspected at the project's beginning. First, I had the opportunity to discuss with my colleagues at the Department of Neurobiology of the Institute of Biophysics in Rio (who are internationally known experts in the different areas of visual research), which particular themes would best illustrate the state-of-the-art. Second, after the difficulty of having to select some of these themes and prospective authors out of a very numerous list, I had the pleasure to receive them all in Rio de Janeiro, and to chair a very active, small meeting held at the Brazilian Academy of Sciences. Third, I enjoyed the privilege of reading all the manuscripts containing scholarly, up-to-date reviews of each theme, as well as original data in some cases. Finally, I was deeply moved with the prospect of dedicating the result of this collective endeavor to the three men who initiated visual research in Rio: Professors Carlos Eduardo Rocha-Miranda, and Eduardo Oswaldo-Cruz, and the laboratory technician Mr. Raymundo F. Bernardes.

The book is organized into two broad sections preceded by an overview. The first section-Genesis - covers many of the numerous fronts of research concerning the developmental biology of the visual system, from either a molecular, a cellular, or a systems angle. The second section-Maturity-takes the same broad approach and covers the structure and physiology of different parts of the visual system, "from photoreceptors to the cortical modules," to quote Pasko Rakic from his Overview.

Both the book and the meeting that preceded it were partially supported by funds obtained from the National Research Council (CNPq), the International Brain Research Organization (IBRO/UNESCO), the Advanced Program of Neuroscience (PAN), the Brazilian Academy of Sciences and IBM-Brazil. The book itself has been competently produced by Birkhäuser Boston.

I hope that we have succeeded in extending to the readers-students, neuroscientists, ophthalmologists, neurologists, and others-the pleasure that we had organizing, discussing, and contributing to the book.

Roberto Lent

Instituto de Biofísica Carlos Chagas Filho 


\section{List of Contributors}

Ruben Adler, Wilmer Ophthalmological Institute, Johns Hopkins University School of Medicine, Baltimore, Maryland, 21205, USA

Michael Ariel, Departments of Behavioral Neuroscience and Psychiatry, University of Pittsburgh, Pittsburgh, Pennsylvania, 15260, USA

Raymundo F. Bernardes, Instituto de Biofísica Carlos Chagas Filho, Universidade Federal do Rio de Janeiro, Centro de Ciências da Saúde, 21941 Rio de Janeiro, Brazil

Richard T. Born, Department of Neurobiology, Harvard Medical School, Boston, Massachusetts, 02115, USA

Hollis T. Cline, Department of Physiology and Biophysics, University of Iowa College of Medicine, Iowa City, Iowa, 52242, USA

Martha Constantine-Paton, Department of Biology, Kline Biology Tower, Yale University, New Haven, Connecticut, 06511, USA

Fernando G. De Mello, Instituto de Biofísica Carlos Chagas Filho, Universidade Federal do Rio de Janeiro, Centro de Ciências da Saúde, 21941 Rio de Janeiro, Brazil

Rodney J. Douglas, Anatomical Neuropharmacology Unit, Medical Research Council, Mansfield Road, Oxford, England

Manuel Esguerra, Department of Brain and Cognitive Sciences, Massachusetts Institute of Technology, Cambridge, Massachusetts, 02139, USA

Rosalia B. Faria, Departamento de Neurobiologia, Universidade Federal Fluminense, Instituto de Biologia, 24001 Niterói, Brazil

Mario Fiorani, Jr., Instituto de Biofísica Carlos Chagas Filho, Universidade Federal do Rio de Janeiro, Centro de Ciências da Saúde, 21941 Rio de Janeiro, Brazil 
Patrícia F. Gardino, Instituto de Biofísica Carlos Chagas Filho, Universidade Federal do Rio de Janeiro, Centro de Ciências da Saúde, 21941 Rio de Janeiro, Brazil

Ricardo Gattass, Instituto de Biofísica Carlos Chagas Filho, Universidade Federal do Rio de Janeiro, Centro de Ciências da Saúde, 21941 Rio de Janeiro, Brazil

Luiz Gawryszewski, Departamento de Neurobiologia, Universidade Federal Fluminense, Instituto de Biologia, 24001 Niterói, Brazil

Charles G. Gross, Department of Psychology, Princeton University, Princeton, New Jersey, 08544, USA

Jong-On Hahm, Department of Brain and Cognitive Sciences, Massachusetts Institute of Technology, Cambridge, Massachusetts, 02139, USA

Jan N. Hokoç, Instituto de Biofísica Carlos Chagas Filho, Universidade Federal do Rio de Janeiro, Centro de Ciências da Saúde, 21941 Rio de Janeiro, Brazil

Roberto Lent, Instituto de Biofísica Carlos Chagas Filho, Universidade Federal do Rio de Janeiro, Centro de Ciências da Saúde, 21941 Rio de Janeiro, Brazil

Rafael Linden, Instituto de Biofísica Carlos Chagas Filho, Universidade Federal do Rio de Janeiro, Centro de Ciências da Saúde, 21941 Rio de Janeiro, Brazil

Marla B. Luskin, Department of Anatomy and Cell Biology, Emory University School of Medicine, Atlanta, Georgia, 30322, USA

Andrew P. Mariani, Neurological Sciences 1, Division of Research Grants, National Institutes of Health, Bethesda, Maryland, 20892, USA

Kevan A. C. Martin, Anatomical Neuropharmacology Unit, Medical Research Council, Mansfield Road, Oxford, England

Rosalia Mendez-Otero, Instituto de Biofísica Carlos Chagas Filho, Universidade Federal do Rio de Janeiro, Centro de Ciências da Saúde, 21941 Rio de Janeiro, Brazil

Eduardo Oswaldo-Cruz, Instituto de Biofísica Carlos Chagas Filho, Universidade Federal do Rio de Janeiro, Centro de Ciências da Saúde, 21941 Rio de Janeiro, Brazil 
Antônio Pereira, Jr., Instituto de Biofísica Carlos Chagas Filho, Universidade Federal do Rio de Janeiro, Centro de Ciências da Saúde, 21941 Rio de Janeiro, Brazil

Cristovam W. Picanço-Diniz, Departamento de Fisiologia, Centro de Ciências Biológicas, Universidade Federal do Pará, 66059 Pará, Brazil

Walter M. Pinheiro, Departamento de Neurobiologia, Universidade Federal Fluminense, Instituto de Biologia, 24001 Niterói, Brazil

Maria C. G. Piñon, Instituto de Biofísica Carlos Chagas Filho, Universidade Federal do Rio de Janeiro, Centro de Ciências da Saúde, 21941 Rio de Janeiro, Brazil

Pasko Rakic, Section of Neurobiology, Yale University School of Medicine, New Haven, Connecticut, 06510, USA

Ary S. Ramoa, Section of Neurobiology, Yale University School of Medicine, New Haven, CT, 06510, and Instituto de Biofísica Carlos Chagas Filho, Universidade Federal do Rio de Janeiro, Centro de Ciências da Saúde, 21941 Rio de Janeiro, Brazil

Giacomo Rizzolatti, Istituto di Fisiologia Umana, Università di Parma, I-43100 Parma, Italy

Carlos E. Rocha-Miranda, Instituto de Biofísica Carlos Chagas Filho, Universidade Federal do Rio de Janeiro, Centro de Ciências da Saúde, 21941 Rio de Janeiro, Brazil

Hillary R. Rodman, Department of Psychology, Princeton University, Princeton, New Jersey, 08544, USA

Marcelo G. P. Rosa, Instituto de Biofísica Carlos Chagas Filho, Universidade Federal do Rio de Janeiro, Centro de Ciências da Saúde, 21941 Rio de Janeiro, Brazil

Burkhard Schlosshauer, Naturwissenschaftliches und Medizinisches Institut, Der Universität Tubingen in Reutlingen, Reutlingen, Germany

Luiz Carlos L. Silveira, Departmento de Fisiologia, Centro de Ciências Biológicas, Universidade Federal do Pará, 66059 Pará, Brazil

Juliana G. M. Soares, Instituto de Biofísica Carlos Chagas Filho, Universidade Federal do Rio de Janeiro, Centro de Ciências da Saúde, 21941 Rio de Janeiro, Brazil 
Aglai P. B. Sousa, Instituto de Biofísica Carlos Chagas Filho, Universidade Federal do Rio de Janeiro, Centro de Ciências de Saúde, 21941 Rio de Janeiro, Brazil

Mriganka Sur, Department of Brain and Cognitive Sciences, Massachusetts Institute of Technology, Cambridge, Massachusetts, 02139, USA

Tania G. Thomaz, Departamento de Neurobiologia, Universidade Federal Fluminense, Instituto de Biologia, 24001 Niterói, Brazil

Roger B. H. Tootell, Department of Neurobiology, Harvard Medical School, Boston, Massachusetts, 02115, USA

Carlo Umiltà, Istituto di Fisiologia Umana, Università di Parma, I-43100 Parma, Italy

Ana L. M. Ventura, Instituto de Biofísica Carlos Chagas Filho, Universidade Federal do Rio de Janeiro, Centro de Ciências da Saúde, 21941 Rio de Janeiro, Brazil

Eliane Volchan, Instituto de Biofísica Carlos Chagas Filho, Universidade Federal do Rio de Janeiro, Centro de Ciências da Saúde, 21941 Rio de Janeiro, Brazil

Edna N. Yamasaki, Instituto de Biofísica Carlos Chagas Filho, Universidade Federal do Rio de Janeiro, Centro de Ciências da Saúde, 21941 Rio de Janeiro, Brazil 